\title{
La paleopalinología: el polen como indicador medioambiental en el sitio Huaca Ventarrón, Lambayeque, Perú
}

Paleopalynology: pollen as an environmental indicator in the Huaca Ventarron, Lambayeque, Perú

DENIS CORREA ${ }^{1}$

\section{RESUMEN}

El material proviene del sitio arqueológico Huaca Ventarrón. Es un tipo de asentamiento ceremonial y está asociado al Formativo Inicial (3000 al 1700 a.C.). La investigación tuvo como objetivo analizar el polen presente en los coprolitos, establecer las condiciones paleoambientales donde se desembolvian estos seres vivos y lograr determinar si es posible emplear los coprolitos de roedores como una fuente fiable de indicadores paleoambientales. En el analisis se emplearon 75 coprolitos de ratones provenientes de las excavaciones arqueológicas; identificándose 18 clases de granos de polen. Se consiguió clasificar taxonómicamente, establecer los restos según el hábito y la comunidad vegetal. Asimismo, el paleoambiente y paleoclima en el cual se desarrollaron estos vegetales. Finalmente fue posible definir que el paisaje natural donde se asentó Huaca Ventarrón está asociado con un entorno boscoso con condiciones climáticas similares a las actuales, lo cual demuestra una estabilidad climática mayor a los 3 mil años.

Palabras clave: huaca ventarrón; coprolito; polen; paleopalinología; arqueobiología.

\begin{abstract}
The material comes from the Huaca Ventarrón archaeological site. It is a type of ceremonial settlement and is associated with the Initial Formative (3000 to $1700 \mathrm{BC}$ ). The objective of the research was to analyze the pollen present in the coprolites, to establish the paleoenvironmental conditions where these living beings were developed and to determine if it is possible to use the rodent coprolites as a reliable source of paleoenvironmental indicators. In the analysis, 75 coprolites of rodents from archaeological excavations were used; identifying 18 classes of pollen grains. It was possible to classify taxonomically, to establish the remains according to the habit and the vegetal community.
\end{abstract}

${ }^{1}$ Université de Rennes. Trujillo, Perú. 
Also, the paleoenvironment and paleoclimate in which these vegetables were developed. Finally, it was possible to define that the natural landscape where Huaca Ventarrón settled is associated with a forested environment with similar climatic conditions to the present, which demonstrates a climatic stability greater than 3 thousand years.

Keywords: huaca ventarron; coprolite; pollen; paleopalynology; archaeobotany.

\section{INTRODUCCIÓN}

En la actualidad se observa un medioambiente variado en toda la costa norte del Perú, con flora y fauna característica según la zona, en otras palabras un dinamismo ecosistémico, que condiciona las actividades económicas de las diversas poblaciones asentadas en lo vasto del territorio costeño. Ante esta realidad, surgen interrogantes relacionadas con la antigüedad de estos entornos ecológicos y sobre el ecosistema presente en épocas pasadas. Estas incógnitas se enfocan directamente en el sitio Huaca Ventarrón, ubicado en el distrito de Pomalca, provincia de Chiclayo, departamento de Lambayeque (figura 01). Teniendo el conocimiento de la importancia social y económica que presenta el sitio de Ventarrón dentro de la costa norte del Perú, surgió la siguiente investigación con el propósito de analizar muestras de coprolito animal de un basural registrado en este sitio arqueológico. La pesquisa tiene como objetivo el análisis paleopalinológico de los granos de polen presente en los coprolitos de roedores (ratones), con la finalidad de establecer las condiciones paleoambientales y paleoclimáticas donde se desenvolvían los restos vegetales identificados.

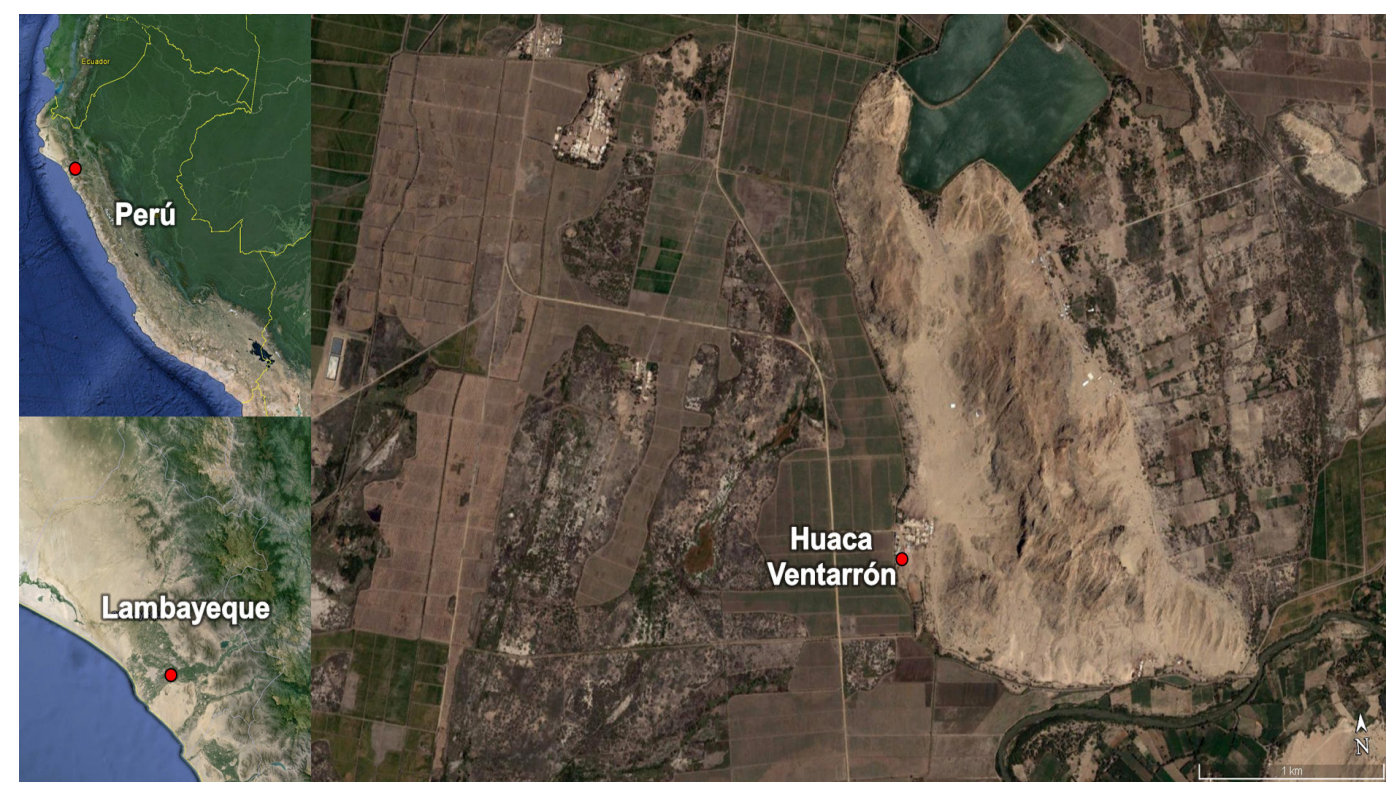

Figura 1. Ubicación política del sitio Huaca Ventarrón (Google Earth, 2017) 


\section{MATERIALES Y MÉTODOS}

El complejo Ventarrón se encuentra conformado por los sitios denominados Huaca Ventarrón y el conjunto Arenal. Huaca Ventarrón está asociado al periodo Formativo Inicial (3000 al 1700 a.C.), pero también presenta entierros intrusivos asociados al periodo Chimú-Inca (Alva, 2008). El sitio se encuentra compuesto por 9 sectores (figura 2) con 10 fases constructivas. Presenta altorrelieve así como murales policromos. Se ha registrado un fogón ceremonial, de cuyas cenizas al ser datadas arrojaron un fechado de 4000 años antes del presente (Alva, 2012).

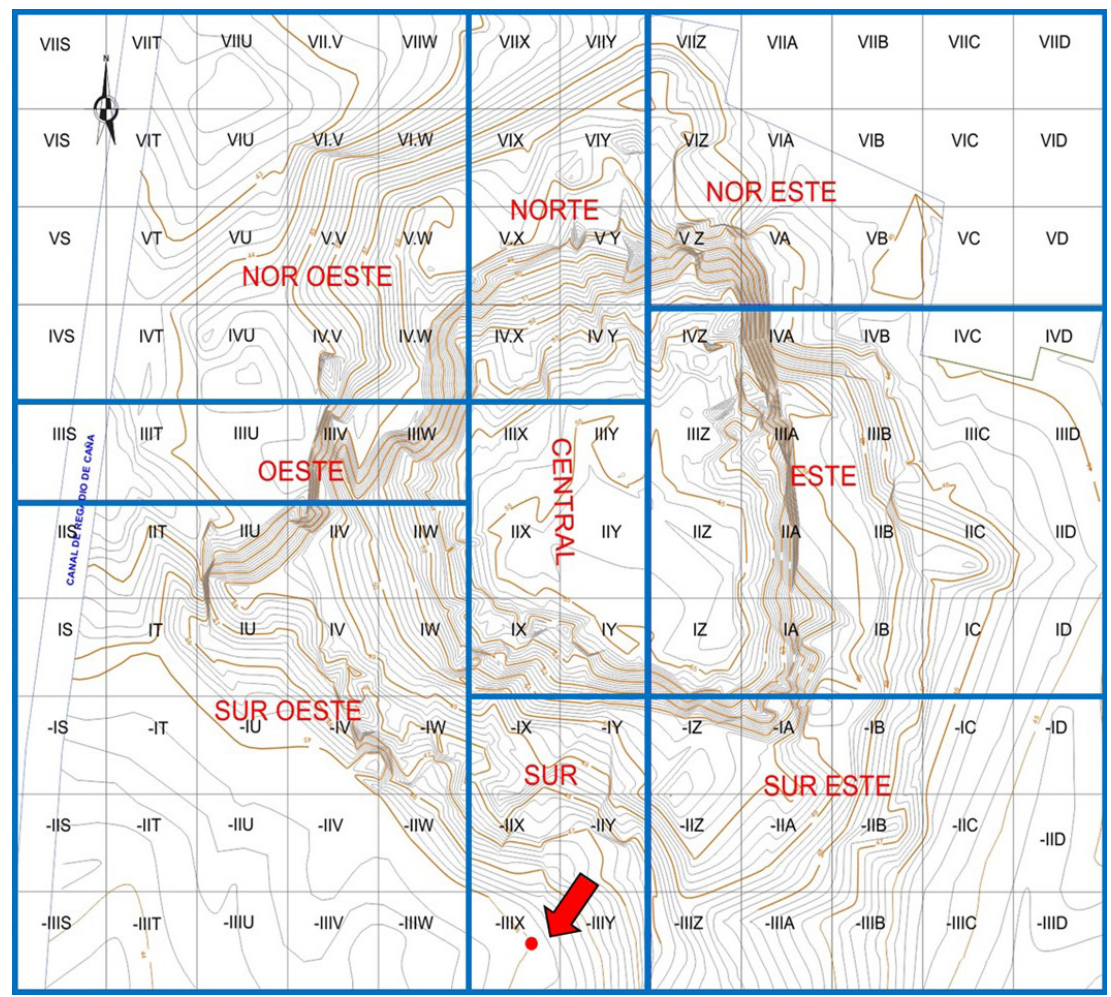

Figura 2. Sectorización del sitio Huaca Ventarrón y la ubicación de la muestra (Alva, 2012:36)

Durante la temporada 2010-2011 (Alva, 2012, 2013), se realizó la excavación de la Unidad -III X y en la parte baja del sector sur se registró el depósito 1a. Este contexto estaría vinculado con los desechos provenientes de las actividades ceremoniales realizadas en el interior del templo y está asociado con la séptima fase de construcción. Se registraron coprolitos, que según su morfología se logró asociar con pequeños roedores (ratones) (figura 3). Asimismo en el mismo contexto se recuperaron diversos materiales culturales como restos de carbones, material lítico, restos óseos de animales (peces y mamíferos), vegetales (semillas) y textiles, los cuales fueron consumidos y utilizados durante la realización de las actividades litúrgicas en el sitio (Vásquez y Rosales, 2012). 


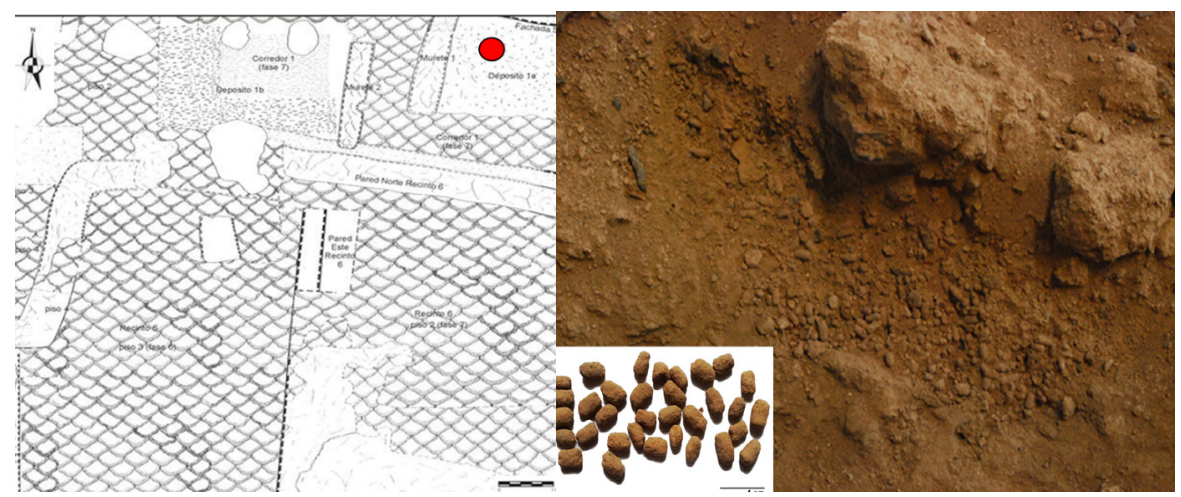

Figura 3. Dibujo de planta de los ambientes asociados con ubicación de los coprolitos en el deposito 1a en la Unidad -III X. La escala corresponde a $1 \mathrm{~m}$ (izquierda) (Alva, 2013). Los Coprolitos de roedores presente en la unidad -IIIX. La escala corresponde a $1 \mathrm{~cm}$ (derecha)

Se ha considerado el uso de los granos de polen debido a su gran resistencia a la degradación química y microbiana (Llergo y Ubere, 2008), la amplitud espacial de dispersión, viento (anemófilos) y animales (zoófilos), que permite estudiar una gran área geográfica adyacente al lugar donde se depositó el polen (Ibarra-Morales y Fernandez-Galán, 2012; Tejero-Diez, Reyes-Salas y Martínez- Hernández, 1988). Asimismo su morfología presenta características diagnosticas que son útiles la identificación taxonómica de familia, género y especie (Canudo, 2002).

En total se recuperaron 332 coprolitos de roedores, los cuales fueron esparcidos homogéneamente sobre la superficie de tres recipientes de $12 \mathrm{~cm}$ x 8 $\mathrm{cm}$. Se seleccionó coprolitos en grupos de 5 de cada uno de los vértices y de la parte central de los recipientes, obteniendo una muestra de 75 coprolitos en total. Los coprolitos restantes se preservaron con la finalidad de tener material para otros tipos de análisis a futuro. El análisis del material coprolital recuperado durante las excavaciones del sitio Huaca Ventarrón tuvo como objetivo la separación e identificación los restos vegetales presentes en los coprolitos analizados. Para este análisis se aplicaron dos metodologías: la rehidratación de coprolitos (Callen y Cameron, 1960) y el aislamiento de granos de polen (Weir y Bonavia, 1985).

Las variedades de granos conservados fueron identificados y clasificados con un microscopio compuesto Olympus modelo CX31 compuesto con un aumento entre 10x, 40x y 100x. Para la identificación de los microrestos se utilizaron las colecciones de referencia del Laboratorio de morfología y fisiología Vegetal y del Herbarium Truxillense, ambos de la Universidad Nacional de Trujillo. El análisis de paleopalinológico se desarrolló en el Laboratorio de morfología y fisiología Vegetal de la Facultad de Ciencias Biológicas, en la Universidad Nacional de Trujillo, Trujillo, Perú. 


\section{RESULTADOS}

En los 75 coprolitos analizados se logró identificar 22 granos de polen (18 determinados y 4 indeterminado), esto permitió obtener información acerca de especies que posiblemente formaban parte de la dieta y actividades económicas de la población local, de igual manera de los factores ambientales del entorno.

\section{Identificación taxonómica}

En el análisis se logró clasificar los granos de polen por orden sistemático y taxonómico, teniendo como resultado 2 divisiones, 3 clases, 11 órdenes, 11 familias, 5 géneros y 2 especies (APGII, 2003, Sáenz Laín, 2004) (tabla 1). Al no lograr determinar 4 tipos de grano de polen debido a su estado de conservación, se le consideró como indeterminado.

Tabla 1. Clasificación sistemática y taxonómica de los restos vegetales registrados en los coprolitos.

\begin{tabular}{cc}
\hline \multicolumn{2}{c}{ DIVISIÓN: MAGNOLIOPHYTA } \\
\hline Orden: Caryophyllales & Orden: Myrtales \\
Familia: Amaranthaceae & Familia: Myrtaceae (Jussieu, 1789) \\
Género: Chenopodium (Linneo, 1753) & Orden: Malvales \\
Orden: Malpighiales & Familia: Malvaceae \\
Familia: Euphorbiaceae (Jussieu, 1789) & Género: Gossypium \\
Orden: Lamiales & Especie: Gossypium barbadense (Linneo, 1753) \\
Familia: Plantaginaceae & Orden: Ericales \\
Género: Plantago (Linneo, 1753) & Familia: Sapotaceae \\
Orden: Oxalidales & Género: Solanum \\
Familia: Oxalidaceae (R. Brown 1818) & Especie: Solanum bycopersicum (Linneo, 1753) \\
CLASE: LILIOPSIDA & Orden: Poales \\
Orden: Asparagales & Familia: Poaceae (Jussieu, 1789) \\
Familia: Amaryllidaceae (Burnett 1835) & DIVISIÓN: ANGIOSPERMAE \\
CLASE: EUDICOTYLEDONEAE & Orden: Ranunculales \\
Orden: Fabales & Familia: Ranunculaceae (Adanson, 1763) \\
Familia: Fabaceae &
\end{tabular}


La presencia de granos de polen en los 75 coprolitos analizados fue relativamente alta y estos presentaron un buen estado de conservación. Según el grupo dentro del cual fueron identificados los restos vegetales, éstos fueron clasificados, de acuerdo al hábito: 12 herbáceas, 10 arbustivas y 6 arbóreas (Mostacero, Mejía y Gamarra, 2009) (tabla 2). Considerando las regiones ecológicas donde se desarrollan las especies de los grupos taxonómicos identificados y la zona de origen de los coprolitos evaluados, se determinó la comunidad vegetal de la costa peruana (Ferreyra, 1983), lográndose inferir el paleoambiente y paleoclima (tabla 03). Se tiene 7 comunidades vegetales, tales como chaparral, ceibal, monte perennifolio y ribereño, algarrobal, gramadal y lomas. Se logró establecer 2 paleoambientes como desértico/ semidesértico, eólico curso efímero; y 1 paleoclima como el árido -semiárido.

Tabla 2. Tipo de vegetación según el hábito y cantidad del contenido vegetal encontrado en los 75 coprolitos analizados

\begin{tabular}{|c|c|c|c|c|c|}
\hline \multirow{2}{*}{\multicolumn{2}{|c|}{$\begin{array}{l}\text { CONTENIDO } \\
\text { VEGETAL }\end{array}$}} & \multicolumn{3}{|c|}{ TIPO DE VEGETACIÓN } & \multirow[t]{2}{*}{ TOTAL } \\
\hline & & HERBÁCEA & ARBUSTIVA & ARBÓREA & \\
\hline \multirow{18}{*}{ 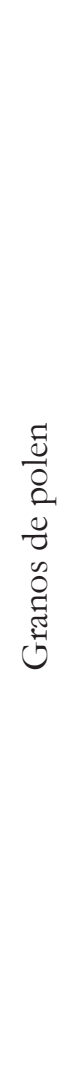 } & Amaranthaceae & $\mathrm{X}$ & $\mathrm{X}$ & & 11 \\
\hline & Amaryllidaceae & $\mathrm{X}$ & & & 3 \\
\hline & Chenopodium sp. & $\mathrm{X}$ & $\mathrm{X}$ & & 14 \\
\hline & Euphorbiaceae tipo 1 & & $\mathrm{X}$ & $\mathrm{X}$ & 4 \\
\hline & Euphorbiaceae tipo 2 & & $\mathrm{X}$ & $\mathrm{X}$ & 2 \\
\hline & Fabaceae & $\mathrm{X}$ & $\mathrm{X}$ & $\mathrm{X}$ & 38 \\
\hline & Phaseolus sp. & $\mathrm{X}$ & & & 1 \\
\hline & Malvaceae & $\mathrm{X}$ & $\mathrm{X}$ & & 1 \\
\hline & Gossypium barbadense & & $\mathrm{X}$ & & 1 \\
\hline & Myrtaceae & & $\mathrm{X}$ & $\mathrm{X}$ & 5 \\
\hline & Oxalidaceae & & $\mathrm{X}$ & $\mathrm{X}$ & 33 \\
\hline & Plantaginaceae & $\mathrm{X}$ & & & 4 \\
\hline & Plantago sp. & $\mathrm{X}$ & & & 1 \\
\hline & Poaceae & $\mathrm{X}$ & & & 19 \\
\hline & Ranunculaceae & $\mathrm{X}$ & & & 4 \\
\hline & Sapotaceae & & & $\mathrm{X}$ & 20 \\
\hline & Solanum sp. & $\mathrm{X}$ & $\mathrm{X}$ & & 4 \\
\hline & Solanum lycopersicum & $\mathrm{X}$ & & & 4 \\
\hline
\end{tabular}


Tabla 3. Comunidad vegetal, paleoambiente y paleoclima de los restos vegetales hallados en los 75 coprolitos analizados.

\begin{tabular}{|c|c|c|c|}
\hline $\begin{array}{l}\text { GRANOS DE } \\
\text { POLEN }\end{array}$ & COMUNIDAD VEGETAL & PALEOAMBIENTE & PALEOCLIMA \\
\hline Amaranthaceae & $\begin{array}{l}\text { Chaparral-Ceibal- Monte } \\
\text { perennifolio-Algarrobal- } \\
\text { Gramadal-Lomas }\end{array}$ & $\begin{array}{l}\text { Desértico/semi- } \\
\text { desértico }\end{array}$ & Árido-semiárido \\
\hline Amaryllidaceae & Lomas & $\begin{array}{l}\text { Desértico/semi- } \\
\text { desértico }\end{array}$ & Árido-semiárido \\
\hline Chenopodium sp. & Gramadal & $\begin{array}{l}\text { Desértico/semi- } \\
\text { desértico }\end{array}$ & Árido-semiárido \\
\hline Euphorbiaceae tipo 1 & Ceibal & $\begin{array}{c}\text { Desértico/semi- } \\
\text { desértico; eólico curso } \\
\text { efímero }\end{array}$ & Árido-semiárido \\
\hline Euphorbiaceae tipo 2 & Ceibal & $\begin{array}{c}\text { Desértico/semi- } \\
\text { desértico; eólico curso } \\
\text { efímero }\end{array}$ & Árido-semiárido \\
\hline Fabaceae & $\begin{array}{l}\text { Ceibal-Algarrobal- } \\
\text { Monte ribereño }\end{array}$ & $\begin{array}{l}\text { Desértico/semi- } \\
\text { desértico }\end{array}$ & Árido-semiárido \\
\hline Phaseolus sp. & $\begin{array}{l}\text { Ceibal-Algarrobal- } \\
\text { Monte ribereño }\end{array}$ & $\begin{array}{l}\text { Desértico/semi- } \\
\text { desértico }\end{array}$ & Árido-semiárido \\
\hline Malvaceae & $\begin{array}{l}\text { Ceibal-Monte perennifolio- } \\
\text { Algarrobal-Lomas- } \\
\text { Monte ribereño }\end{array}$ & $\begin{array}{l}\text { Desértico/semi- } \\
\text { desértico }\end{array}$ & Árido-semiárido \\
\hline Gossypium barbadens & Ceibal & $\begin{array}{l}\text { Desértico/semi- } \\
\text { desértico }\end{array}$ & Árido-semiárido \\
\hline Myrtaceae & Lomas & $\begin{array}{c}\text { Desértico/semi- } \\
\text { desértico; eólico curso } \\
\text { efímero }\end{array}$ & Árido-semiárido \\
\hline Oxalidaceae & Ceibal & $\begin{array}{c}\text { Desértico } / \text { semi- } \\
\text { desértico; eólico curso } \\
\text { efímero }\end{array}$ & Árido-semiárido \\
\hline Plantaginaceae & Ceibal & $\begin{array}{l}\text { Desértico/semi- } \\
\text { desértico }\end{array}$ & Árido-semiárido \\
\hline Plantago sp. & Ceibal & $\begin{array}{l}\text { Desértico/semi- } \\
\text { desértico }\end{array}$ & Árido-semiárido \\
\hline Poaceae & $\begin{array}{l}\text { Manglar-Chaparral- } \\
\text { Algarrobal-Gramadal-Lomas }\end{array}$ & $\begin{array}{l}\text { Desértico/semi- } \\
\text { desértico }\end{array}$ & Árido-semiárido \\
\hline Ranunculaceae & Monte ribereño & $\begin{array}{l}\text { Desértico/semi- } \\
\text { desértico }\end{array}$ & Árido-semiárido \\
\hline Sapotaceae & Ceibal & $\begin{array}{l}\text { Desértico/semi- } \\
\text { desértico } \\
\text { eólico curso efímero }\end{array}$ & Árido-semiárido \\
\hline Solanum sp. & Algarrobal-Lomas & $\begin{array}{l}\text { Desértico/semi- } \\
\text { desértico }\end{array}$ & Árido-semiárido \\
\hline Solanum lycopersicum & Algarrobal-Lomas & $\begin{array}{l}\text { Desértico/semi- } \\
\text { desértico }\end{array}$ & Árido-semiárido \\
\hline
\end{tabular}




\section{DISCUSIÓN}

Producto de los análisis se logró determinar además de las condiciones tanto paleoclimáticas y paleoambientales la dieta de los roedores productores de los coprolitos. Se logró determinar dos especies: Solanum lycopersicum "tomate" y Gossypium barbadense "algodón”, de igual manera tres géneros: Chenopodium sp., Phaseolus sp. y Plantago sp., vinculados con vegetales utilizados en la dieta, industria (León, 2013) y medicina (Mostacero, Mejía y Gamarra, 2009). La combinación alimenticia entre plantas silvestres y cultivadas es coherente con el tipo de contexto en donde se registró, así como el comportamiento natural del roedor, ya que al estar en contacto directo con el ser humano se genera un estado de "comensalismo", en el cual los ratones sacan beneficio del hombre mediante el consumo de sus alimentos o desperdicios; asimismo ocupando las estructuras con la finalidad de lograr obtener un refugio y calor. En estos entornos de abundancia, los ratones se inclinan a seleccionar una dieta nutricionalmente balanceada cuando se le da a elegir entre un amplio rango de alimentos diferentes (Benavides y Guénet, 2003). Este comportamiento se encuentra completamente evidenciado por que el tipo de contexto donde se registraron los coprolitos es de un depósito de basura que contenía restos orgánicos proveniente de las áreas internas del templo (ambientes) y de las actividades dentro de la misma (ceremonias y mantenimiento) (Alva, 2012).

Con respecto al clima presente durante la ocupación del sitio Huaca Ventarrón, se logró establecer que según el tipo de flora identificada, correspondería a un paleoclima árido-semiárido, de similares condiciones climáticas que las actuales (López, 1986). De tal forma se determinó que este clima tiene una permanencia mayor a los 3 mil años, considerando la datación de 2300-2035 a.C. que tiene la primera fase constructiva registrada en el sitio, lo cual concuerda con el registro polínico en múltiples áreas de Sudamérica que establece una estabilidad climática de alrededor de unos 4 mil años (Mc Glone, Kershaw y Markgraf, 1992; Villagrán, 1993).

En relación al paleoambiente en donde se asentaron la población de Huaca Ventarrón, se considera que por la cantidad de granos de polen indicaría la presencia de un nivel riqueza vegetal relativamente alta en la zona adyacente. Asimismo la totalidad de los grupos taxonómicos identificados se desarrollan en ambientes xéricos (desértico/semi-desértico), lo cual concuerda con las condiciones ambientales actuales en la zona (INDECI, 2003) y corrobora la información paleoclimática establecida. 
Lamentablemente alrededor del sitio se conserva muy poca flora natural en la actualidad, siendo los campos de cultivos los que predomina el paisaje. Según el tipo de vegetación identificadas y las características tanto paleoambientales como paleoclimaticas se puede establecer que durante el desarrollo pleno de sus actividades, el entorno estuvo dominado por hierbas, arbustos y vegetación leñosa natural, un entorno similar a las condiciones identificadas en Huaca Ventarrón sería el Santuario Histórico Bosque de Pómac, en donde es posible reconocer los diversos paleoambientes determinados en la presente investigación (Angulo, 2013; SENARP, 2011).

\section{CONCLUSIONES}

Los resultados demuestran la gran utilidad del polen presente en los coprolitos de roedores como registros de la vegetación del pasado e indicador paleoambiental, ya que, se logró obtener información acerca de aspectos medioambientales de la zona, de los distintos vegetales que formaban parte de la dieta, tanto de los roedores como los pobladores de Huaca Ventarrón. Igualmente de las actividades económicas de la población local y las comunidades vegetales aledañas.

Asimismo, se ha logrado determinar que durante la ocupación de este asentamiento el paisaje natural era muy diferente al actual, mayor presencia de bosque seco, pero con similares condiciones medioambientales a las actuales. Esto se logró determinar ya que las especies vegetales identificadas se desarrollaron en un paleoambiente desértico/ semidesértico y con un paleoclima árido-semiárido. Estas características aún se encuentran presentes en la región, como demuestra una estabilidad climática desde hace alrededor 3 mil años.

\section{AGRADECIMIENTOS}

Al director del Proyecto de Investigación Arqueológica Ventarrón - Collud: Ignacio Alva Meneses, quien me brindó todas las facilidades para realizar el análisis de los coprolitos de roedores. A los doctores Santiago Uceda Castillo y Ramiro J. March por sus esfuerzos para desarrollar la maestría y abrir los horizontes de muchos jóvenes investigadores y Dra Mercedes E. Chaman Medina, responsable del laboratorio de morfología y fisiología Vegetal de la Facultad de Ciencias Biológicas, en la Universidad Nacional de Truji1lo. A mi amigo y colega Eduardo Eche Vega, por las correcciones del texto. 


\section{REFERENCIAS BIBLIOGRÁFICAS}

Alva, Ignacio, 2008. «Los complejos de Cerro Ventarrón y Collud-Zarpán: del Precerámico al Formativo en el valle de Lambayeque». Boletín de Arqueología PUCP, Vol. 12, Lima: Departamento de Humanidades, Pontificia Universidad Católica del Perú. 97-117.

Alva, Ignacio. 2012. Ventarrón y Collud. Origen y auge de la civilización en la costa norte del Perú. Lambayeque: Ministerio de Cultura, Proyecto Especial Naylamp Lambayeque.

Alva, Ignacio, 2013. Proyecto de Investigación Arqueologica Ventarrón-Collud. Informe de la Temporada 2010-2011. Lambayeque: Proyecto Especial Naylamp Lambayeque- Unidad Ejecutora $N^{\circ} 005$.

Angulo, Fernando. 2013. Guía de Aves Santuario Histórico Bosque de Pómac. Lima: Ministerio de Comercio Exterior y Turismo.

APGII (Angiosperm Phylogeny Group), 2003. «An update of the Angiosperm Phylogeny Group classification for the ordens and families of flowering plants: APG II». Botanical Journal of the Linnean Society, $\mathrm{N}^{\circ} 141$, Londres: The Linnean Society of London. 339-436.

Benavides, Fernando y Guénet, Jean-Louis, 2003. Manual de genética de roedores de laboratorio. Principios básicos y aplicaciones. Madrid: Universidad de Alcalá de Henares.

Callen, Eric y Cameron, Thomas, 1960. «A prehistoric diet revealed in coprolites». The new scientist, Vol. 8, $\mathrm{N}^{\circ}$ 190, Londres: Reed Business Information. 35-40.

Canudo, José, 2002. Polen y esporas, Eustoquio, Molina (editor) Micropaleontología. Universidad de Zaragoza, Zaragoza: 347-363.

Ferreyra, Ramón, 1983. «Los tipos de vegetación de la costa peruana». Anales del Jardín Botánico de Madrid, Vol. 40, N 1. Madrid: Royal Botanic Gardens. 241-256.

Ibarra- Morales, Emilio y Fernández-Galán, Beatriz, 2012. «El estudio del polen antiguo: Problemas y estrategias en el laboratorio». Revista Especializada en Ciencias Químico-Biologicas, Vol. 15, № 1. Ciudad de México: Universidad Autónoma de México. 62-66.

INDECI (Instituto Nacional de Defensa Civil), 2003. Mapa de peligros de la ciudad de Lambayeque. Lambayeque: Proyecto INDECI-PNUD PER/02/051, Ciudades sostenibles. 
Mc Glone, Matt; Kersiiaw, Peter y Markgraf, Vera, 1992. «El Niño/ Southern Oscillation climatic variability in Australasian and South American paleoenvironmental record». En Díaz, Henry y Markgraf, Vera (editores). El Niño; Historical and Paleoclimatic Aspects of the Southern Oscillation, Cambrige: Cambrige Univeristy Press. 243-258.

Mostacero, José; Mejía, Freddy y Gamarra, Oscar. 2009. Fanerógamas del Peru: Taxonomía, utilidad y ecogeografía. Trujillo: CONCYTEC.

León, Elmo. 2013. 14,000 años de alimentación en el Perú. Lima: Universidad San Martín de Porres.

Llergo, Yolanda y Ubera, José, 2008. «Cambios en el entorno vegetal de Carmona (Sevilla) desde el Calcolítico hasta el Medievo». Carel, $\mathrm{N}^{\circ}$ 6, Carmona: Ayuntamiento de Carmona. 2369-2393.

López, Carlos, 1986. «Desertificación en el noroeste costero del Perú. Zonas aridas». Centro de investigación de Zonas Áridas. Lima: Universidad Nacional Agraria. 32-40.

Sáenz Laín, Concepción. 2004. «Glosario de términos palinológicos». Lazaroa $\mathrm{N}^{\circ}$ 25. Madrid: Universidad Complutense de Madrid. 93-112.

SENARP (Servicio Nacional de Áreas Protegidas por el Estado), 2011. Plan Maestro Santuario Histórico Bosque de Pómac. Lima: Servicio Nacional de Áreas Naturales Protegidas por el Estado.

Tejero-Diez, Daniel; Reyes-Salas, Margarita y Martínez-Hernández Enrique, 1988. «Lluvia de polen moderno, en un gradiente altitudinal con vegetación templada en el municipio de Ocuilan, Estado de Mexico, Mexico». Palinologica et Paleobotanica, 1, México DF: 61-80.

Vásquez, Víctor y Rosales, Teresa, 2012. «Restos de fauna y vegetales de Huaca Ventarrón: Unidad - IIIX». En Alva, Ignacio (editor). Ventarrón y Collud. Origen y auge de la civilización en la costa norte del Perú. Lambayeque: Ministerio de Cultura, Proyecto Especial Naylamp Lambayeque. 251-272.

Villagrán, Carolina, 1993. «Una interpretación climática del registro palinológico del último ciclo glacial- postglacial en Sudamérica». Bulletin de l'Institut Français d'Etudes Andines, Vol. 22, No 1. Lima: Instituto Francés de Estudios Andinos. 243-258. 
|Denis correa

Weir, Glendon y Bonavia, Duccio, 1985. «Coprolitos y dieta del precerámico Tardío de la costa peruana». Bulletin de l'Institut Français d'Etudes Andines Vol. 34, N 1-2. Lima: Instituto Francés de Estudios Andinos. 85-140.

Presentado: 06/02/2017

Aceptado: 16/05/2017

Coorrespondencia

Denis Correa

denis_1627@hotmail.com 